\title{
A Vivência de Práticas Físicas/Motoras/Esportivas de Homens e Mulheres para Propor o Construto Orientação Esportiva
}

http://dx.doi.org/10.11606/1807-5509202000030395
Fernando Luiz Cardoso* Thiago Emannuel Medeiros* Walan Robert Da Silva* Elisa Pinheiro Ferrari* Gislane Ferreira de Melo ${ }^{* *}$
*Universidade do Estado de Santa Catarina, Florianópolis, SC, Brasil.

**Universidade Católica de Brasília, Brasília, DF, Brasil.

\section{Resumo}

0 objetivo deste trabalho teórico-empírico foi investigar o histórico de vida das práticas físicas/motoras/ esportivas de 407 graduandos de Ed. Física de uma universidade pública (211 homens e 196 mulheres) para tentar averiguar um traço de orientação para a prática de diferentes modalidades esportivas. Esse estudo foi realizado a partir de dados empíricos sobre a experiência prévia dos seus participantes para identificar um possivel traço latente comum aos sujeitos desta pesquisa em suas preferências esportivas. $\mathrm{Na}$ análise dos dados utilizou-se recursos da estatística descritiva e inferencial por meio de testes $t$ de student, qui quadrado, análise de regressão linear (backward) e análise fatorial exploratória. Os resultados mostraram que homens quanto as mulheres gostam tanto de atividades físicas cíclicas como complexas. No entanto, os homens demonstraram gostar mais de atividades físicas complexas. A diferença em relação ao perfil motor/esportivo entre os sexos caracteriza-se pela preferência dos homens por esportes coletivos de embate, enquanto as mulheres, por atividades físicas coletivas ligadas ao ritmo. Diante dos resultados observados foi possível identificar um traço predominante para homens e mulheres, embasando a proposta de um novo constructo sobre a orientação esportiva composto por duas dimensões, embatividade e ritmicidade. Este novo constructo exige a proposição de um instrumento de medida que possa medir e predizer a orientação esportiva nas Ciências do Movimento.

Palavras-Chave: Educação Física; Sexo; Orientação Motora; Prática Esportiva; Orientação Esportiva.

\section{Introdução}

O processo de identificação e escolha de práticas esportivas em atletas geralmente se dá muito cedo $^{1}$, porém pouco se sabe sobre quais aspectos eliciam a escolha dos indivíduos por diferentes modalidades esportivas. Um fenômeno que parece ter pouco respaldo em termos comportamentais é o constructo de orientação esportiva ${ }^{2,3}$.

Partindo da psicometria tradicional, o desenvolvimento do conhecimento geralmente segue as seguintes etapas: teorias, conceitos, construtos $^{4}$, as quais nem sempre são seguidas nas áreas de Educação Física e Psicologia do Esporte para produção de conhecimento. TROCHIM ${ }^{5}$ por sua vez, afirma que existem duas formas básicas de se elaborar a um novo construto a partir de: uma teoria robusta ou do comportamento observável. Segundo o mesmo autor, as teorias são as concepçôes provenientes da mente do pesquisador, enquanto o comportamento se configura em aquilo que pode ser observado sobre o fenômeno de interesse.

O conceito de orientação esportiva mais conhecido e utilizado pela comunidade acadêmica é o questionário de Gill e DeEter ${ }^{6}$, o qual é composto por três fatores psicológicos, a competitividade, o desejo de entrar e se esforçar para o sucesso na competição esportiva, orientação para a vitória caracterizada pelo foco em padróes de relacionamento interpessoal e de vitória e, orientação para a meta, foco em padrões pessoais. 
Neste sentido, identifica-se que a compreensão da orientação para o esporte está baseada nas características da personalidade do indivíduo.

Diferentemente de Gill e DeETER ${ }^{6}$, o presente estudo adotou a concepção de elaboração de constructo para a orientação esportiva baseado em Trochim ${ }^{5}$, o qual sugere as experiências vividas pelos indivíduos para propor um traço de comportamento e sustentar um constructo. No atual estudo, o comportamento estudado para fundamentar a elaboração do traço latente na orientação esportiva se baseará nas práticas físicas /motoras/ esportivas vivenciadas e recordadas pelos indivíduos.

Estas práticas físicas podem ser compreendidas a partir da sua organização e complexidade, isto é, por meio da sua estrutura e dificuldade. NAYLOR e BRIGGS $^{7}$ define como complexidade, uma função de demandas no processamento de informações imposta por cada componente da atividade separadamente, e organização as exigências, interações ou inter-relaçõos entre os componentes da atividade. Assim, a complexidade da tarefa é uma característica intra componente, e a sua organização uma característica entre os componentes.

CARDOSO $^{8}$ propõe que estas atividades físicas/ motoras/ esportivas podem ser descritas e classificadas a partir de algumas especificidades, como, por exemplo, à sua natureza (mais ou menos organizadas e complexas) e pelo seu nível de interação (grupo ou individualizada). O mesmo autor pontua a questão da preferência por determinados tipos de atividades podendo as mesmas serem pensadas sob a ótica de uma orientação psicomotora especifica, em termos de potencialidade que as pessoas possuam ou construam, como a embatividade (atividades com embate e grande contato físico) e a ritmicidade (atividades que envolvem ritmo, musicalidade e dança) motora.

Assim, os indivíduos demonstram diferentes preferências por atividades físicas e jogos de forma geral, podendo ser essas atividades de alta organização - maior simetria de suas partes constituintes proporcionando um maior encadeamento das partes do movimento (natação, ciclismo), atividades de alta complexidade - maior assimetria das partes do movimento (futebol, ginástica). Essas preferências poderiam suscitar uma orientação para o esporte a partir da natureza e tipo de modalidade que o indivíduo costuma ou tem preferência em praticar. Com isso, surgem as perguntas norteadoras deste estudo: $\mathrm{O}$ histórico da vivência de práticas físicas e motoras prediria o gosto ou a orientação por estas atividades esportivas citadas acima? A orientação esportiva poderia se basear no agrupamento de modalidades para indicação um traço de orientação? A orientação esportiva apresentaria alguma influência dos tradicionais estereótipos sociais de gênero definidos socialmente para homens e mulheres?

A partir dessas perguntas acredita-se que a proposição de um novo constructo e de um futuro instrumento de medida para a orientação esportiva possa ser utilizado no processo de iniciação esportiva em escolas e clubes com o objetivo de detectar traços que melhor encaminhem as nossas crianças na experimentação da grande diversidade de opçóes esportivas oferecidas.

Assim sendo, a identificação de um ou mais traços motores comuns a praticantes de diferentes modalidades esportivas poderiam nos ajudar a classificar e predizer o novo construto aqui proposto, a orientação esportiva para uma ou mais modalidades. Nesse contexto, o objetivo deste foi estudar o histórico de práticas físicas\motoras esportivas de graduandos de um curso de Educação Física buscando listar, comparar e identificar traços de orientação esportiva para uma determinada modalidade ou agrupamento delas. A partir desses dados acredita-se ser possível a formulação de um novo construto para a orientação esportiva baseado nas experiências motoras na infância e não nas tradicionais teorias psicológicas.

\section{Método}

Esta é uma pesquisa qualitativa e quantitativa não probabilística que buscou evidências comportamentais para elaborar um novo constructo para a orientação esportiva baseado em escolhas físicas $\backslash$ motoras $।$ esportivas feitas por alunos de graduação em Educação
Física ao longo de suas vidas. Os procedimentos empíricos para elaboração do novo constructo foram aprovados pelo Comitê de Ética em Pesquisa da UDESC, sob o número de protocolo 40/05 e estão descriminados na FIGURA 1 abaixo. 


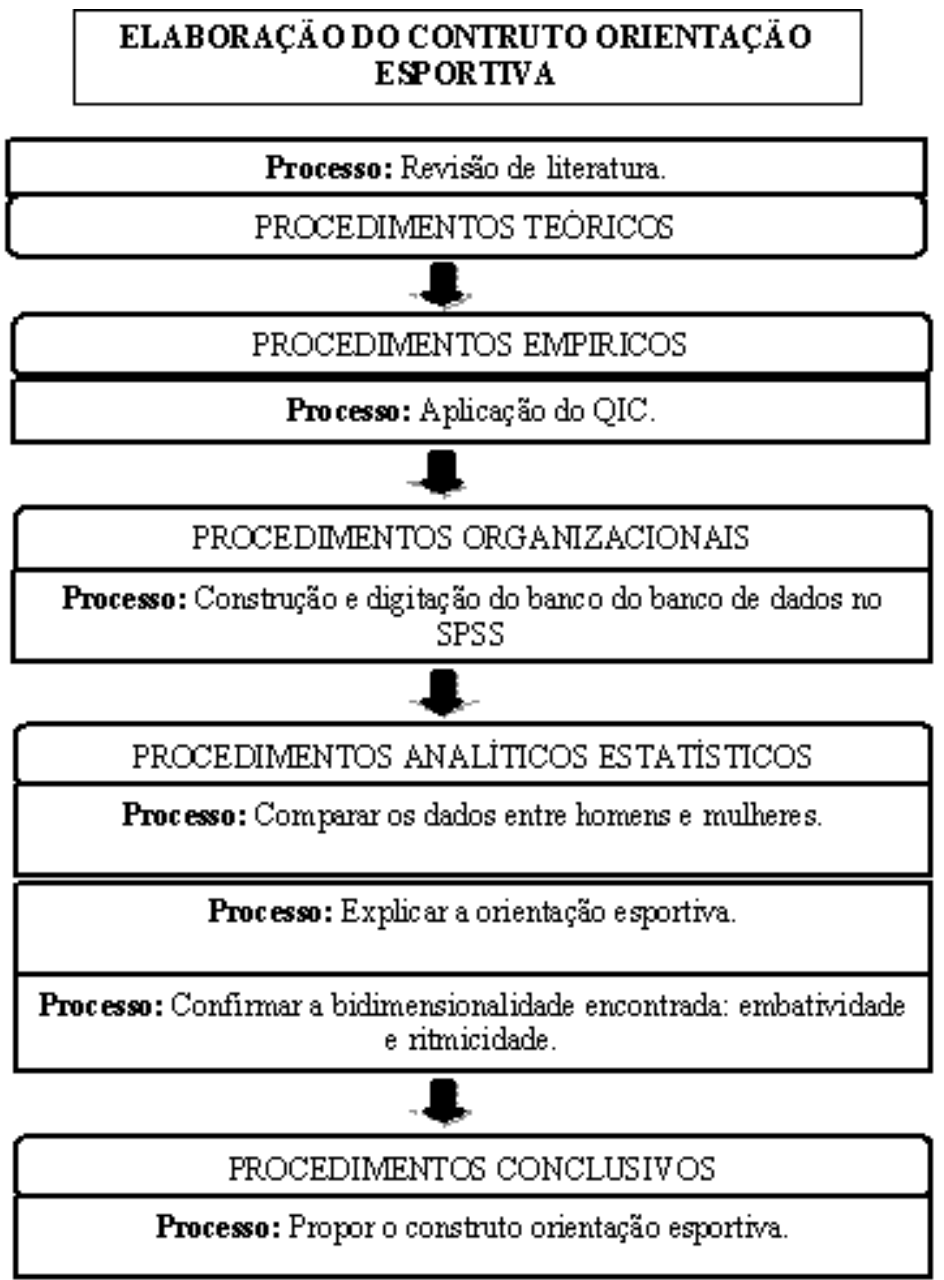

FIGURA 1 -Etapas metodológicas para a elaboração e proposição do construto orientação esportiva.

\section{Participantes}

Participaram do estudo 407 estudantes, sendo 211 homens e 196 mulheres, matriculados regularmente em um curso de graduação em Educação Física. Os alunos foram selecionados aleatoriamente e convidados em sala de aula a responderem ao questionário. Ninguém se negou a participar da pesquisa.

Em relação ao perfil sócio antropométrico dos participantes do estudo, foram observadas diferenças significativas na estatura e massa corporal entre homens e mulheres, como esperado. Além disso, o nível de atividade física entre os homens apresentaram valores superiores às mulheres (TABELA 1).

\section{Instrumento e coleta de dados}

Utilizou-se os itens da dimensão corporeidade do Questionário de Identidade Corporal (QIC), desenvolvido pelo Laboratório de Gênero, Sexualidade e Corporeidade do CEFID UDESC como um instrumento diagnóstico para caracterizar diferentes populações na pesquisa. O QIC é constituído de 85 itens distribuídos em três dimensões: corporeidade, sexualidade e esportividade e já serviu de base para criação de outros instrumentos de medida ${ }^{9}$. Nesse estudo concentrou-se nos itens relativos as dimensões corporeidade e esportividade.

\section{Análise dos dados}

Os dados empíricos foram organizados no programa estatístico SPSS versão 20.0, utilizando recursos da estatística descritiva (média, desvio padrão e frequência), e inferencial por meio dos testes $t$ de student para amostras independentes, Qui-quadrado para diagnosticar diferenças significativas entre homens e mulheres, e análise de regressão linear com extração backward 
para saber o efeito das variáveis independentes sobre a variável dependente preferência por atividades complexas. Aplicou-se o teste de normalidade de Kolmogorov Smirnov para todas as variáveis. Além disso, realizou-se a análise fatorial exploratória para verificação do agrupamento de práticas psicomotora-esportivas. $\mathrm{O}$ valor de significância adotado foi de $\mathrm{p}<0,05$.

TABELA 1 -Perfil sócio antropométrico dos homens e mulheres participantes.

\begin{tabular}{|c|c|c|c|c|c|c|}
\hline \multirow{2}{*}{ Parâmetros } & \multicolumn{2}{|c|}{ Homens } & \multicolumn{2}{|c|}{ Mulheres } & \multirow{2}{*}{ t test } & \multirow{2}{*}{$P$} \\
\hline & $X$ & $s d$ & $X$ & $s d$ & & \\
\hline Idade & 22,17 & 3,46 & 21,70 & 3,68 & 1,17 & 0,24 \\
\hline \multirow[t]{2}{*}{ Nível de atividade física* } & 3,62 & 1,77 & 2,80 & 1,66 & 4,55 & 0,01 \\
\hline & \multicolumn{2}{|c|}{$\mathrm{n}(\%)$} & \multicolumn{2}{|c|}{$\mathrm{n}(\%)$} & $X^{2}$ & $P$ \\
\hline $\begin{array}{l}\text { Prática atividade física } \\
\text { regularmente** }\end{array}$ & & & & & 2,39 & 0,03 \\
\hline $\operatorname{Sim}$ & \multicolumn{2}{|c|}{$134(66,5)$} & \multicolumn{2}{|c|}{$113(5,7)$} & & \\
\hline Não & \multicolumn{2}{|c|}{$77(36,5)$} & \multicolumn{2}{|c|}{$82(41,8)$} & & \\
\hline
\end{tabular}

Sig. (2-tailed) $\leq 0,05 ;{ }^{*} \mathrm{O}$ quanto ativo você se considera em termos de atividade física? Média de uma escala de sete pontos onde 0 seria nada ativo e o 6 muito ativo; ** Atualmente você pratica exercício físico regularmente (pelo menos 01 hora no mínimo de 2 vezes por semana)?

\section{Resultados}

Em relação ao universo de práticas de atividades físicas, motoras e esportivas dos nossos participantes, 134 homens (66,5\%) e 113 mulheres $(57,7 \%)$ disseram praticar atividade física regularmente. Os fisicamente ativos apontaram as seguintes atividades esportivas como as preferidas, relatadas na TABELA 2. Outro aspecto observado refere-se a predominância das distintas práticas entre os sexos com relação às modalidades esportivas, das quais os homens em geral praticam futebol, basquetebol, rúgbi e artes marciais que são modalidades de alto contato físico. Já as mulheres praticam mais modalidades físicas como caminhada, ginástica, ballet, dança e voleibol, pois, são atividades mais calmas e sem contato físico. Dessa maneira, observa-se que homens e mulheres assumiram práticas esportivas distintas, sendo que, $37,8 \%$ dos homens e $45,4 \%$ das mulheres praticam atividades físicas sem embate corporal, enquanto $21,8 \%$ dos homens e $7,1 \%$ das mulheres praticam atividades físicas com embate corporal.

TABELA 2 -Diferentes práticas esportivas realizadas regularmente ou eventualmente pelos homens e mulheres participantes.

\footnotetext{
* Questão aberta: Qual atividade física você pratica regularmente?
}

\begin{tabular}{lcccc}
\hline $\begin{array}{l}\text { Pratica de Ativida- } \\
\text { des esportivas* }\end{array}$ & Homens & \multicolumn{2}{c}{ Mulheres } \\
\cline { 2 - 5 } & $\mathbf{n}$ & $\mathbf{\%}$ & $\mathbf{n}$ & $\mathbf{\%}$ \\
\hline Futebol & 26 & 12,3 & 09 & 4,6 \\
Basquetebol & 04 & 1,9 & 00 & 0,0 \\
Handebol & 02 & 0,9 & 01 & 0,5 \\
Rúgbi & 04 & 1,9 & 00 & 0,0 \\
Voleibol & 01 & 0,5 & 03 & 1,5 \\
Remo & 04 & 1,9 & 00 & 0,0 \\
Natação & 06 & 2,8 & 07 & 3,6 \\
Musculação & 33 & 15,6 & 34 & 17,3 \\
Ginástica & 04 & 1,9 & 08 & 4,1 \\
Atletismo & 03 & 1,4 & 00 & 0,0 \\
Artes marciais & 09 & 4,3 & 04 & 2,0 \\
\hline
\end{tabular}

continua 
continuação

\begin{tabular}{lcccc}
\hline \multirow{2}{*}{$\begin{array}{c}\text { Pratica de Ativida- } \\
\text { des esportivas* }\end{array}$} & Homens & \multicolumn{2}{c}{ Mulheres } \\
\cline { 2 - 5 } Caminhada & $\mathbf{n}$ & $\mathbf{0}$ & $\mathbf{n}$ & $\mathbf{\%}$ \\
Corrida & 00 & 0,0 & 10 & 4,1 \\
Ciclismo & 11 & 5,2 & 08 & 1,5 \\
Hóquei & 06 & 2,8 & 03 & 0,0 \\
Dança & 01 & 0,5 & 00 & 8,7 \\
Surf & 01 & 0,5 & 17 & 0,0 \\
Yoga & 10 & 4,7 & 00 & 1,0 \\
Várias Atividades & 01 & 0,5 & 02 & 0,0 \\
Total & 05 & 2,4 & 00 & $\mathbf{1 0 0}, 0$ \\
\hline
\end{tabular}

Em relação às preferências físicas, verificouse na TABELA 3 que homens e mulheres têm similaridades por práticas motoras cíclicas, porém os homens têm ligeira preferência por práticas motoras complexas. A partir disso, comparou-se então a preferência por atividades físicas cíclicas e complexas entre homens e mulheres e não foram observadas diferenças entre atividades cíclicas como andar, correr, andar de bicicleta e nadar $(\mathrm{p}<0,05)$. No entanto, mais homens preferem atividades físicas complexas, como futebol e capoeira $(p<0,001)$. Devido a esses resultados, decidiu-se analisar com maior profundidade as atividades complexas preferidas por homens e mulheres. Além disso, cabe destacar a maior preferência dos homens por atividades complexas, bem como, por artes marciais, capoeira, futebol e basquete. Estas modalidades possuem em sua natureza características de alta complexidade e embate físico o que pode influenciar a preferência dos homens pelas mesmas. Já as mulheres apresentaram uma maior preferência por ginástica, atividades físicas com ritmo e música, dança de salão, dança de rua, yoga e ballet. Pode se observar, que as mulheres preferem atividades de natureza mais cíclica em que a realização dos movimentos exige pouco ou nenhum embate físico, bem como necessita de ritmo durante a execução.

TABELA 3 -Perfil da orientação psicomotora esportiva dos homens e mulheres participantes.

\begin{tabular}{|c|c|c|c|c|c|c|}
\hline \multirow{2}{*}{ Variáveis } & \multicolumn{2}{|c|}{ Homens } & \multicolumn{2}{|c|}{ Mulheres } & \multirow{2}{*}{ t test } & \multirow[b]{2}{*}{$p$} \\
\hline & $X$ & $s d$ & $X$ & $s d$ & & \\
\hline Gosta de atividades físicas mais complexas & 4,19 & 1,99 & 2,93 & 2,13 & 6,14 & 0,001 \\
\hline Gosta de fazer artes marciais & 2,40 & 2,20 & 1,48 & 1,92 & 4,42 & 0,001 \\
\hline Gosta de fazer capoeira & 1,64 & 1,97 & 1,09 & 1,68 & 2,96 & 0,003 \\
\hline Gosta de jogar futebol & 4,08 & 2,12 & 2,47 & 2,23 & 7,39 & 0,001 \\
\hline Gosta de jogar basquete & 2,80 & 2,09 & 2,03 & 1,92 & 3,79 & 0,001 \\
\hline Gosta de fazer ginástica & 1,85 & 1,74 & 3,45 & 1,99 & $-8,56$ & 0,001 \\
\hline Gosta de atividade física com música & 2,72 & 1,92 & 4,43 & 1,68 & $-9,42$ & 0,001 \\
\hline Gosta de fazer dança de salão & 1,77 & 1,90 & 3,08 & 2,43 & $-5,96$ & 0,001 \\
\hline Gosta de fazer dança de rua & 1,36 & 1,81 & 2,54 & 2,30 & $-5,60$ & 0,001 \\
\hline Gosta de fazer yoga & 1,27 & 1,81 & 2,00 & 2,13 & $-3,60$ & 0,001 \\
\hline Gosta de fazer ballet & 0,32 & 0,91 & 1,48 & 2,98 & $-6,54$ & 0,001 \\
\hline
\end{tabular}

Com o objetivo de buscar uma compreensão sobre a orientação esportiva para atividades físicas complexas para ambos os sexos foi realizada uma regressão linear com extração backward. Das 19 variáveis independentes relacionadas, para ambos os sexos $\left(\mathrm{R}^{2}=.529\right)$, se o sujeito gosta de atividades físicas em grupo $(\beta=.182 ; \mathrm{IC}, .115$ a $.338, \mathrm{p}<.001)$, se o sujeito gosta de esportes radicais $(\beta=.098$; IC, 
.003 a .184, $\mathrm{p}<.043)$, se o sujeito gosta de jogar futebol( $\beta=.435$; IC, .288 a $.456, \mathrm{p}<.001)$, se o sujeito gosta de jogar handebol $(\beta=.104$; IC, .011 a .177, $\mathrm{p}<.026)$, se o sujeito gosta de fazer artes marciais $(\beta=.114$; IC, .016 a .187, $\mathrm{p}<.020)$ e se o sujeito gosta de fazer capoeira $(\beta=.169$; IC, .069 a $.268, \mathrm{p}<.001)$, explicam de forma significativa $52,9 \%$ da orientação motora para atividades físicas complexas entre homens e mulheres.

Por fim, na TABELA 4 foi realizada a análise fatorial exploratória com o Método de extração, com Análise do Componente principal em dois fatores, em que o Método de rotação: Oblimin com normalização de Kaiser $(0,827)$ indicou na Matriz de estruturas o agrupamento de modalidade/ práticas esportivas em esportes de ritimicidade e embatividade para amostra investigada. Todos os itens com cargas fatoriais acima de 0,30. Os itens que se referenciavam as práticas de capoeira e yoga foram os únicos que não carregaram com direcionamento nas duas dimensôes percebidas nas análises anteriores.

TABELA 4-Distribuição dos itens da escala nas duas dimensões propostas: Ritimicidade e Embatividade.

\begin{tabular}{|c|c|c|}
\hline \multirow{2}{*}{ Itens } & \multicolumn{2}{|c|}{ Componentes } \\
\hline & Embatividade & Ritimicidade \\
\hline Se o sujeito faz atividades mais complexas como futebol e capoeira & ,863 & \\
\hline Se o sujeito faz atividades físicas em grupo & ,814 & \\
\hline Se o sujeito joga futebol & ,795 & \\
\hline Se o sujeito joga basquetebol & ,746 & \\
\hline Se o sujeito faz atividades físicas individualmente & ,728 & \\
\hline Se o sujeito pratica artes marciais & ,709 & \\
\hline Se o sujeito faz mais atividades cíclicas, como caminhada e ciclismo & ,598 & \\
\hline Se o sujeito faz esportes de aventura ou radicais & ,570 & \\
\hline Se o sujeito joga voleibol & &, 801 \\
\hline Se o sujeito faz ginástica & & ,766 \\
\hline Se o indivíduo faz dança & & ,752 \\
\hline Se o sujeito faz Yoga & & ,681 \\
\hline Se o sujeito faz dança de salão & & ,677 \\
\hline Se o sujeito faz atividades físicas que tenham ritmo ou música & & ,646 \\
\hline Se o sujeito faz musculação & 653 &, 526 \\
\hline Se o sujeito nada & ,605 &, 553 \\
\hline Se o sujeito gostade de atividades físicas mais complexas como: futebol e capoeira & ,737 & \\
\hline Se o sujeito gosta de esportes radicais & 670 & \\
\hline Se o sujeito gosta de esportes de aventura & ,655 & \\
\hline Se o sujeito gosta de jogar futebol & 650 & \\
\hline Se o sujeito gosta de jogar basquete &, 588 & \\
\hline Se o sujeito gosta de fazer capoeira &, 570 & \\
\hline Se o sujeito gosta de jogar handebol &, 553 & \\
\hline Se o sujeito gosta de fazer artes marciais &, 548 & \\
\hline Se o sujeito gosta de jogar vôlei & ,497 & \\
\hline Se o sujeito gosta de fazer dança de rua & & ,752 \\
\hline Se o sujeito gosta de atividades físicas com ritmo ou música & & ,735 \\
\hline Se o sujeito gosta de fazer dança de salão & & ,706 \\
\hline Se o sujeito gosta de fazer ginástica & & ,706 \\
\hline Se o sujeito gota de fazer ballet & & 669 \\
\hline Se o sujeito gosta de fazer yoga & &, 566 \\
\hline Se o sujeito gosta de nadar & ,328 & ,311 \\
\hline
\end{tabular}




\section{Discussão}

Dentre os sujeitos investigados, os homens apresentaram-se fisicamente mais ativos e praticantes de um maior número de atividades físicas em relação às mulheres, dados esses já aferidos pela literatura ${ }^{10}$. Segundo PIERON ${ }^{11}$ isso ocorre devido a maior importância que os homens dão a sensação de serem fisicamente competentes, o que acaba por levá-los a uma participação mais atuante em atividades físicas e no esporte de forma geral, quando comparados às mulheres. No mesmo sentido CARDOSo et al. ${ }^{12}$ demonstram em seu estudo que homens em geral se percebem mais ativos fisicamente e gostam mais de atividades que buscam competitividade e agressividade do que as mulheres. Tais achados segundo os mesmos autores nos levam a pensar na importância da agressividade e embatividade na formação da masculinidade.

No que tange a preferência por atividades físicas, segundo GALLAHUE e OZMUN ${ }^{13}$ a preferência por atividades físicas e esportivas podem estar relacionadas à iniciação esportiva ou a fase de conscientização para a prática de atividades físicas, em que os indivíduos experimentam diferentes tipos de atividades motoras tanto no ambiente escolar como fora dele. CARDOSO e SACOMORI ${ }^{9}$ mostraram que mulheres atletas geralmente tiveram uma infância próxima de meninos o que provavelmente despertou o interesse destas pela pratica do treinamento desportivo. Infelizmente não se controlou nesse estudo a identidade de gênero do ponto de vista cognitivo, que classificaria a identidade de gênero entre masculinidade, androgenia e feminilidade tanto para homens como para mulheres. Pois sabemos que nem todo o homem ou mulher tem o mesmo nível de masculinidade ou feminilidade em termos de comportamento ${ }^{1}$.

O presente estudo traz dados relevantes nesse sentido, em que os homens preferem modalidades esportivas com maior competitividade e embate corporal como futebol, basquetebol, rúgbi e artes marciais, já as mulheres optam por modalidades voltadas as ginásticas e dança em que não ocorrem embates corporais e sua realização é mais cooperativa que outras modalidades, essa preferência distinta entre homens e mulheres pode ser pensada com relação aos tradicionais estereótipos de gênero vigentes na sociedade $\mathrm{em}^{7}$ que os homens buscam atividades com maior risco no qual a força e o combate são as características principais ${ }^{14}$, e as mulheres buscam atividades mais calmas, mais cooperativas, com ritmo e música ${ }^{15}$. Tais escolhas poderiam indicar um esforço de ambos os sexos em interpretar e incorporar adequadamente os estereótipos esperados para cada sexo, recebendo gratificações sociais destes comportamentos adequados.

Os resultados do estudo de CARDOSO et al. ${ }^{12}$ também demonstrou essa tendência por atividades menos competitivas e agressivas para as mulheres que poderiam ser traduzidos pelo receio social destas em corromper a sua identidade feminina ao praticar atividades esportivas "masculinas" não adequadas as expectativas sociais. No mesmo sentido, estudo realizado pelo Instituto de La Mujer da Espanha em 2005 ${ }^{16}$, as atividades preferidas entre elas foram a natação, ginástica aeróbica e a ginástica de manutenção, demonstrando dessa maneira a preferência média das mulheres por atividades mais cíclicas e sem embate físico.

Por fim, uma possível explicação para essa tendência e gosto dos homens por atividades agressivas e de embate físico pode ser pensada com relação à herança filogenética, onde a agressividade e o embate físico foram aspectos importantes para os machos ganharem mais status social e obterem mais vantagens reprodutivas ${ }^{17}$.

A partir do momento que observamos as diferentes preferências de homens e mulheres por algumas modalidades, cabe-se pensar que a natureza das atividades pode ser um fator que influencie essa preferência ${ }^{5}$. No presente estudo, isso é observado a partir de que os homens demonstraram diferenças com relação às mulheres em gostar de modalidades como futebol, basquetebol, capoeira, artes marciais e atividades físicas mais complexas, modalidades essas caracterizadas por uma constante variação dos seus movimentos, em que o indivíduo deve ter cinestesia as assimetrias e nuances motoras para vivenciá-las.

Segundo Silva, Halperni e Silva ${ }^{18}$, essa maior preferência por atividades complexas entre os homens pode ter influenciado a maior participação desses no que diz respeito a pratica de atividades como futebol, basquetebol, artes marciais, capoeira e esportes radicais e de aventura que requer maior atenção ou concentração. Essa preferência já relatada em estudos de alguns autores ${ }^{19}$ pode estar 
relacionada aos estímulos que são oferecidos aos homens desde a infância, como maior liberdade motora, por exemplo, o que em consequência traria a estes uma motricidade mais rica.

Já as mulheres do presente estudo apresentaram uma preferência por atividades como ginástica, atividades físicas com ritmo e música, dança de salão e de rua, yoga e ballet, modalidades essas com mais características cíclicas e sem embate corporal, ou seja, essas atividades privilegiam a execução dos movimentos de forma fechada, esperada e controlada. Essa preferência por atividades mais cíclicas e com música vai ao encontro do estudo de Moura et al. ${ }^{15}$, em que a música torna-se fator motivacional durante aulas de bike indoor, o que também segundo CARDOSO e SACOMORI ${ }^{9}$ por serem atividades mais individualizadas, calmas e de menor contato físico leva a uma maior participação das mulheres pelo ambiente mais acolhedor.

Com relação, à experiência motora, no estudo de Cardoso et al. ${ }^{12}$ com homens e mulheres atletas, os praticantes de futebol faziam mais atividades físicas complexas e em grupo, e os nadadores praticavam mais esportes de aventura ou radicais e modalidades individuais, ilustrando dessa forma um perfil ou um traço do indivíduo para atividades com estruturas similares.

Os dois grandes aspectos diferenciadores em relação ao constructo, orientação esportiva foram: a embatividade, percebida pelos pesquisadores como, práticas em que ocorre choque, encontro, pancada, resistência ou oposição entre os praticantes e ritmicidade motora entendida pelos pesquisadores como atividades ritmadas, compassadas ou com cadência normalmente acompanhada por música.

A ideia de orientação esportiva apresentada por Gill e DeETER ${ }^{6}$ caracteriza-se por ênfase nos traços de personalidade (competitividade, orientação para a vitória e orientação para meta). A identificação dessas duas dimensôes (embatividade e ritmicidade esportiva) nesse estudo sustentada por dados empíricos define alguns aspectos talvez inatos. Como também confirma uma das dimensões psíquicas sugeridas por GILl e DEETER ${ }^{6}$, a competitividade. $\mathrm{O}$ mais importante é ressaltar que embora os homens tenham ligeira preferência por atividades complexas, não foi a natureza cíclica e complexa que diferenciou homens e mulheres, mas sim a presença ou ausência das duas dimensões: embatividade e ritmicidade nas atividades cíclicas e complexas.
Dessa maneira, sugere-se no presente estudo o entendimento de orientação esportiva a partir da preferência por atividades de embate e de ritmo propondo-se assim uma forma de graduarmos a orientação esportiva que tem a capacidade de distinguir as diferentes preferências físicas e motoras dos indivíduos, discriminando algumas particularidades predominantes para cada sexo.

PASQUALI ${ }^{4}$ aponta que a teoria deve fundamentar qualquer empreendimento científico e, com isso, deve ser clara a explicitação da teoria sobre a qual o teste irá ser construído. Nesse sentido, acredita-se que esse intendo logrou êxito em descrever, analisar e discriminar os reais aspectos comportamentais que influenciam as preferências por práticas físicas e motoras de homens e mulheres, mostrando que a natureza cíclica ou complexa da atividade explica pouco, sendo a presença ou ausência de embate físico e ritmicidade os fatores diferenciadores.

Ao estudar o histórico de vida dos participantes observou-se que homens em geral têm práticas motoras distintas das mulheres. Ao analisar essas diferenças percebeu-se homens e mulheres gostam de atividades físicas cíclicas e os homens têm ligeira preferência por atividades complexas. Somente a natureza complexa das atividades físicas não explica as preferências de homens e mulheres, mas dois componentes constitutivos da atividade física: embatividade e ritmicidade esportiva, em que homens em geral têm maior preferência pelo primeiro e as mulheres em geral pelo segundo, dimensões estas confirmadas pela análise fatorial.

Este estudo limitou-se e estudar o histórico da vivencia das atividades físicas $\backslash$ motoras $\backslash$ esportivas de estudantes de um curso de Educação Física, aspectos psicológicos, como a identidade de gênero cognitivo não foram controlados. Assim acreditase que essas diferenças encontradas entre os sexos, pode não se restringir apenas ao sexo biológico, isto é, independentemente de ser homem ou mulher, a identidade de gênero (masculina, feminina e androgenia), o perfil psicológico de gênero (heteroesquemático masculino, heteroesquemático feminino e isoesquemático) poderiam também explicar a preferência pela embatividade ou ritimicidade.

Os autores do manuscrito informam não haver conflitos de interesse e assumem que estão trabalhando na construção e testagem de um Instrumento de Avaliação da Orientação Esportiva humana a partir do constructo aqui apresentado e defendido. 


\begin{abstract}
The objective of this theoretical-empirical work was to study the life history of the physical, motor and sports practices of 407 undergraduate Physical Education students from a public university $(211$ men and 196 women) to try to identify a guideline for different sports modalities' practice. Data analysis was performed using resources of descriptive and inferential statistics using the Student t Test, Chi Square, Linear Regression Analysis (backward) and Exploratory Factorial Analysis. The results showed that both sexes like cyclical and complex physical activities, however, men like more complex physical activities than women. The difference from physic-motor-sport profile between the sexes is characterized by preference of men for clash/contact team sports, while women, by collective physical activities related to the rhythm. So that it was possible to identify a predominant trait for men and women that based on the proposal of a new construct on the sports orientation based on two dimensions that can being applied to both sexes: combativeness and rhythmicity. This new construct requires the proposition of an evaluation instrument that can measure and predict the sports orientation in the Sciences of the Human Movement.
\end{abstract}

KeYwords: Physical Education; Sex; Motor Orientation; Sports Practice; Sport Orientation.

\title{
Referências
}

1. Malina RM.Talent Identification and Selection in Sport. Rio de Janeiro: [editora desconhecida]; 2000.

2. Filin PV, Volkov VM. Selecção de Talentos no Desporto. Londrina: Midiograf; 1998.

3. Estriga MLD. Orientação Desportiva: em crianças e jovens nas modalidades de Andebol, basquetebol e voleibol [Dissertação]. Porto: Universidade do Porto; 2000.

4. Pasquali L. Princípios de elaboração de escalas psicológicas. Rev Psiquiatr. 1998;25:206-213.

5. Trochim WMK. Research Methods Knowledge Base. Sydney: Conjoint.ly; 2006. Introduction to Validity.

6. Gill DL, Deeter TE. Development of the sport orientation questionnaire. Res Q Exerc Sport. 1988;3:191-202.

7. Naylor JC, Briggs GE. Effects of task complexity and task organization on the relative efficiency of part and whole training methods. J Exp Psychol. 1963;65:217-224.

8. Cardoso FL. O conceito de orientação sexual na encruzilhada entre sexo, gênero e motricidade. Interam J psychol. 2008;42:69-79.

9. Cardoso FL, Sacomori C. Identidade de gênero/sexo de atletas e sedentários. Rev Bras Ciênc Esporte. 2012;34:925941.

10. Mendes M, Melo V, Mares V, Silva C, Moreira P, Santos Júnior W, et al. Percepção e prática de atividade física regular entre usuários de serviço de atenção primária à saúde. Rev APS. 2013;16:151-7.

11. Pieron M. Estilo de vida, prática de atividades físicas e esportivas, qualidade de vida. Fit Perform J. 2004;3:10-17.

12. Cardoso FL, Silveira RA, Zequinão MA, Martins C, Souza CA. Auto-percepção corporal e preferências motoras de praticantes de dança. Movimento. 2009;16:97-112.

13. Gallahue DL, Ozmun JC. Compreendendo o desenvolvimento motor: bebês, crianças, adolescentes e adultos. São Paulo: Phorte; 2001.

14. Pellegrini AD, Smith PK. Physical activity play: The nature and function of a neglected aspect of play. Child Dev. 1998;69:577-98.

15. Moura NL, Grillo DE, Merida M, Campanelli JR, Merida F. A influência motivacional da música em mulheres praticantes de ginástica de academia. Rev Mackenzie de Educ Fís e Esp. 2009;6:103-118.

16. Instituto De La Mujer. III Plan de Igualdad de Oportunidades entre Mujeres y Hombres de España (1997-2000). Madrid: Instituto de la Mujer; 1997.

17. Wrangham R, Peterson D. O Macho Demoníaco: as origens da agressividade humana. Rio de Janeiro: Objetiva; 1998. 
Cardoso FL, et al.

28. Silva CAD, Halperni FBSC, Silva LAD. Meninas bem-comportadas, boas alunas; meninos inteligentes, indisciplinados. Cad Pesq. 1999;107:207-225.

19. Cardoso FL, Marinho A, Pimentel GGA. Questóes de gênero em universitários praticantes de esportes de aventura. Rev Educ Fis UEM. 2013;24(4):597-608.

ENDEREÇO DE CORRESPONDÊNCIA:

Fernando Luiz Cardoso

Rua Benvenuta James, 69 - Centro

Florianópolis - SC - BRASIL

CEP: 88015-630

E-mail: fernandocardoso.ph.d.lagesc@gmail.com

Submetido: 07/07/2015

Revisado: 16/11/2016

Aceito: 28/11/2017 